\title{
Warning Signs of Tipping Point for Greenland Ice Sheet
}

\author{
A stability analysis of the ice sheet covering a large area of Greenland \\ suggests that the ice melting may be approaching a point of no return.
}

\author{
By Richard Blaustein
}

$\prod$ he Greenland ice sheet-an important component of Earth's climate system-is losing mass at an accelerating rate. A great concern is that the ice sheet could be heading toward a tipping point, beyond which the ice cap would be permanently lost. A new study, published in the Proceedings of the National Academy of Science, suggests that a large part of Greenland's ice sheet may be close to such a tipping point. The complete melting of Greenland's ice cap could lead to a sea-level rise of up to $7 \mathrm{~m}$ and may trigger a cascade of other climate instabilities.

Climate tipping points are thresholds beyond which large-scale, sudden changes in Earth's climate system occur.

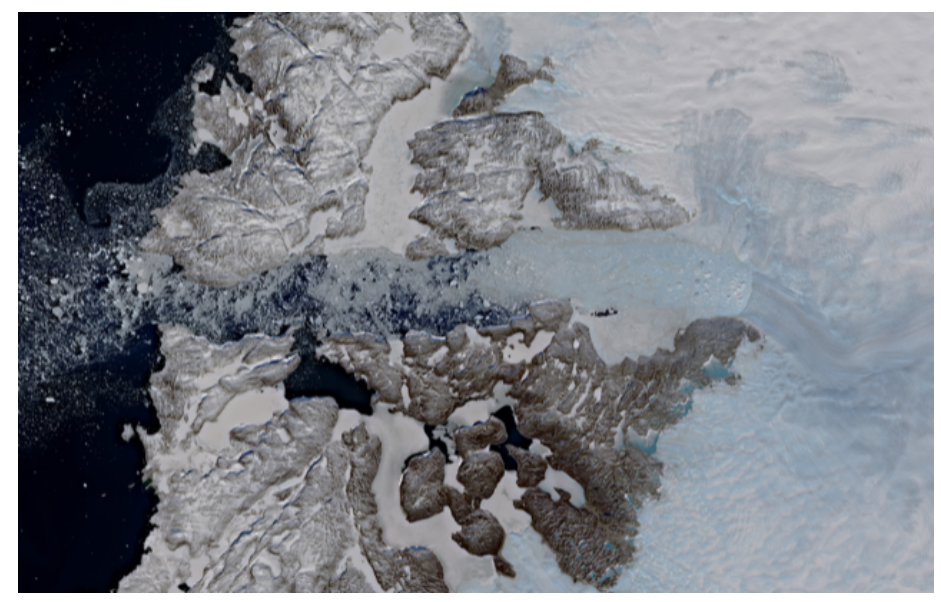

Satellite image of the Jakobshavn Glacier-the fastest retreating glacier in Greenland. The analysis of melt rates in the last 150 years indicates the glacier might be approaching a tipping point. Credit: Copernicus Sentinel data, processed by ESA
Paleogeological records have revealed that such tipping has occurred in the past-examples include the desertification of Sahara and abrupt temperature changes during the last ice age. But current climate models, such as those used by the Intergovernmental Panel on Climate Change, have had difficulties in elucidating past climactic changes, calling into question the ability of these models to predict future tipping.

A promising approach is given by nonlinear dynamics theories, which offer powerful tools for describing the temporal evolution of natural systems. Previous studies based on nonlinear dynamics suggested that there may be precursor signs of an approaching tipping point, associated with a slowing down of the system's dynamical behavior.

Physicist Niklas Boers of the Potsdam Institute for Climate Impact Research in Germany and mathematician Martin Rypdal of the University of Tromsø - Arctic University of Norway have now found these "critical slowing down" signals for the Jakobshavn Glacier-the fastest melting of Greenland's ice basins. The duo obtained their result by applying nonlinear dynamics theory to analyze 150 years' worth of data on the glacier's melt rates, inferred from ice-core samples.

Their study focuses on one of the crucial elements governing the dynamics of the Greenland ice sheet: a feedback mechanism, called melt-elevation feedback, that connects melting with ice elevation. Melting reduces the ice-sheet height, so the ice gets exposed to the warmer air found at lower elevations, which, in turn, accelerates melting.

Boers says that a key advantage of using sheet heights, rather 


\section{RESEARCH NEWS}

than melt rates, as a model parameter is that height is a "state variable"-much like temperature or pressure. As such, sheet height provides a clearer indication of whether the system might be close to the phase transition associated with climate tipping, he says. Indeed, the sheet-height history revealed signs of critical slowing down.

Critical slowing down occurs when a system approaches a bifurcation that makes its current equilibrium state unstable. Under such conditions, the system takes longer to return to equilibrium following a perturbation than it would if the system was in a stable state. Critical slowing down can be indicated by two parameters describing the fluctuations of the system. The variance is the magnitude of the fluctuations around the equilibrium, and it increases close to the bifurcation because the forces that drive the system toward equilibrium weaken. Autocorrelation quantifies how much the system's state at a given time is similar to the state at a previous time. For a system on its way to a tipping point, a slowed-down recovery means that this similarity increases, yielding a large autocorrelation value.

For the Jakobshavn Glacier, the researchers found that the autocorrelation approaches unity, and the variance is diverging-both suggestive of an approaching tipping point. The researchers acknowledge that more research is needed to firmly establish whether a tipping point has already been reached or whether it might be close. In particular, other feedback mechanisms need to be considered, including both destabilizing effects-notably, the decrease of albedo due to ice-cover loss-and stabilizing effects-such as the increase of snowfall due to warming temperatures.

Alexander Robinson, a climate scientist and glaciologist at the Complutense University of Madrid, says these results will help future modeling. And while the results do not necessarily apply to the entire ice sheet covering Greenland, he thinks that studying the Jakobshavn Glacier region is particularly important. "You could call it the 'weak underbelly' of the Greenland ice sheet," Robinson says. "It is where you'll likely get the most warming and [ice] retreat. So, it might be the place to see changes earliest."

"The results are sobering, but the way they have managed to [obtain them] is impressive," says University of Exeter, UK, climate scientist Tim Lenton. While the ice sheet is a sluggish system that changes on very slow timescales, the fact that the researchers could extract signatures of the slowed-down fluctuations from observational data is "a real pleasant surprise." Lenton says that results like those obtained by Boers and Rypdal support earlier calls for developing tools for anticipating climate change impacts (see, for instance, the 2013 National Academy of Sciences report). "With a relatively modest investment we could develop an early warning signal for a range of climate tipping points," Lenton says.

Richard Blaustein is a freelance science and environmental journalist based in Washington, DC. 